\title{
OPEN Cage bedding modifies metabolic and gut microbiota profiles in mouse studies applying dietary restriction
}

\author{
A. Gregor ${ }^{1}$, L. Fragner ${ }^{2,3}$, S. Trajanoski ${ }^{4}$, W. Li ${ }^{3}$, X. Sun ${ }^{3}$, W. Weckwerth ${ }^{2,3}$, J. König ${ }^{1,3}$ \& \\ K. Duszka ${ }^{1,3 凶}$
}

Experiments involving food restriction are common practice in metabolic research. Under fasted conditions, mice supplement their diet with cage bedding. We aimed at identifying metabolic and microbiota-related parameters affected by the bedding type. We exposed mice housed with wooden, cellulose, or corncob cage beddings to ad libitum feeding, caloric restriction (CR), or over-night (ON) fasting. Additionally, two subgroups of the $\mathrm{ON}$ fast group were kept without any bedding or on a metal grid preventing coprophagy. Mice under CR supplemented their diet substantially with bedding; however, the amount varied depending on the kind of bedding. Bedding-related changes in body weight loss, fat loss, cecum size, stomach weight, fecal output, blood ghrelin levels as well as a response to glucose oral tolerance test were recorded. As fiber is fermented by the gut bacteria, the type of bedding affects gut bacteria and fecal metabolites composition of CR mice. CR wood and cellulose groups showed distinct cecal metabolite and microbiome profiles when compared to the CR corncob group. While all ad libitum fed animal groups share similar profiles. We show that restrictionrelated additional intake of bedding-derived fiber modulates multiple physiological parameters. Therefore, the previous rodent studies on CR, report the combined effect of CR and increased fiber consumption.

It is commonly agreed that mice need to be housed with bedding and nesting material to fulfill the animals' welfare requirements. Cage bedding is an important factor for animal well-being. It provides nesting material, helps to keep warmth, provides a proper walking surface, and buffers air ammonia content ${ }^{1}$. However, mice and rats fed at ad libitum and, even more, under dietary restrictions tend to consume cage bedding ${ }^{2}$. Moreover, multiple metabolic tests involving rodents are preceded by over-night $(\mathrm{ON})$ fasting. Thus, the type of bedding and mice's ability to extract energy from the bedding will impact the results of the metabolic tests. An even bigger impact is expected in the case of caloric restriction (CR). CR requires daily delivery of a limited, accurately dosed amount of food. In animal as well as in human studies CR has been shown to increase lifespan and health-span. It prevents the development of various diseases including age-related, neurological, and metabolic diseases as well as cancer ${ }^{3,4}$. For a successful CR experimental protocol, mice energy intake has to be strictly adjusted. Thus, uncontrolled energy uptake and fiber supplementation by consuming cage bedding can substantially influence the experimental outcome. Accordingly, in our previous publication ${ }^{5}$, we showed that mice submitted to CR develop an increased cecum size. We concluded that the enlarged cecum results from the accumulation of indigestible fiber as a consequence of bedding consumption. The enlarged cecum is observed upon consumption of a high-fiber $\operatorname{diet}^{6-8}$ but it is also a phenotype typical to germ-free mice and indicates aggregation of fiber mass as well as disturbed nitrogen reabsorption in the small intestine ${ }^{9-11}$. Importantly, fiber can also reduce nutrient digestibility as it limits the access of enzymes to nutrients and it may also affect the passage rate of the digesta, especially if consumed in high amounts ${ }^{12}$. Accordingly, it has been noticed that feed conversion is reduced from animals housed on corncob (CC) bedding ${ }^{13}$. Two previous publications addressed the issue of the impact of the $\mathrm{CC}$ bedding on mice body weight and gut microbiota concluding that application of the CC bedding may

\footnotetext{
${ }^{1}$ Department of Nutritional Sciences, University of Vienna, Althanstrasse 14 (UZA II), 1090 Wien, Austria. ${ }^{2}$ Molecular Systems Biology, Department of Functional and Evolutionary Ecology, University of Vienna, Vienna, Austria. ${ }^{3}$ Vienna Metabolomics Center, University of Vienna, Vienna, Austria. ${ }^{4}$ Core Facility Computational Bioanalytics, Medical University of Graz, Graz, Austria. ${ }^{\circledR}$ email: kalina.duszka@univie.ac.at
} 
confound study results ${ }^{13,14}$. However, no one, thus far, compared different types of bedding considering metabolic research and microbiota.

Bedding consumption is particularly important in the context of the rapidly developing field of gut microbiota. Dietary fibers, including those present in cage bedding, may undergo complete or partial fermentation by the gut microbes ${ }^{15}$, leading to the production of short-chain fatty acids (SCFA) and stimulating the growth of certain bacterial species ${ }^{16}$. The metabolic end products of colonic microbiota play an important role in the maintenance of health and the development of disease ${ }^{15,17}$. Succeeding inconsistencies in scientific outcomes, the issue of reproducibility in microbiota research was inevitably raised ${ }^{18,19}$. We aim at studying the variability in research outcomes introduced by cage beddings as well as bringing awareness to the reproducibility of the studies involving fasting and CR by stressing the importance of bedding.

\section{Results}

Cage bedding affects mice body and organ weight. To assay the impact of cage bedding we submitted mice to two kinds of dietary restrictions in different housing conditions. The mice were held with one of the cage beddings: wooden (W), cellulose (C), or corncob (CC). The animals from over-night fasted (ON) groups were housed with one of three cage beddings (ON-W, ON-C, ON-CC) and additional groups without bedding (no bedding, ON-NB) or on a metal grid (ON-G). The grid was preventing the animals from contact with the cage bottom, thus limiting coprophagy. In parallel, to assess the long-term effects of bedding consumption we submitted mice to 14 days CR using the three kinds of cage bedding: wooden (CR-W), cellulose (CR-C), and corncob (CR-CC). Corresponding control groups (W, C, CC) were housed ad libitum with one of the assigned cage beddings. The mice housed with wooden bedding ate the biggest amount of bedding while mice on cellulose bedding ate the least of bedding (all groups $\mathrm{p}<0.001$; Fig. 1a). With similar starting bodyweight (Supplementary Fig. S1), overnight-fasted mice from ON-W, ON-C, ON-CC, ON-NB groups lost 13-14\% body weight (Fig. 1b) while ON-G mice lost $16 \%$ body weight suggesting the impact of coprophagy and/or cage bedding consumption on body weight. CR mice lost $20-22 \%$ body weight within 14 days with the CR-CC group losing the most. At the same time, all ad libitum mice gained 6-8\% body weight. There were no differences in the weight of the stomach with its content between the ad libitum and CR groups (Fig. 1c) indicating comparable consumption in hours prior to the dissection, even though CR mice had access to food for the last time the evening preceding the dissection. Mice from all ON groups had lighter stomachs than ad libitum fed and CR mice proving diminished consumption ( $\mathrm{W}$ vs $\mathrm{ON}-\mathrm{W} \mathrm{p}=0.004, \mathrm{C}$ vs ON-C p=0.006). Both ON-G (ON-W vs ON-G p $=0.002$, ON-C vs ON-G p<0.001, ON-CC vs ON-G p<0.001) and ON-NB (ON-CC vs ON-NB $p=0.002)$ groups had smaller stomachs than other ON groups suggesting the lowest food intake. Among ON fasted groups, CC group had the heaviest stomach and its content implying increased consumption or slower digestibility of CC bedding compared to $\mathrm{W}$ and C. CR mice, which experienced long-lasting dietary restriction, had heavier stomach with its content than $\mathrm{ON}$ mice suggesting that long-term food restriction enhances supplementation with cage bedding and/or feces. Mice from all CR groups showed an increased weight of cecum with its content compared to ad libitum mice (Fig. 1d) pointing toward an accumulation of indigestible fiber. The cecum of the CR-CC group was significantly smaller than the cecum of CR-W mice $(p=0.005)$ suggesting less fiber deposition. All ON fast mice showed decreased cecum weight compared to ad libitum mice. ON-G group had a statistically significantly lighter cecum than any other ON mice (ON-G vs ON-W p $<0.01$, ON-G vs ON-C p $<0.01$, ON-G vs ON-CC $\mathrm{p}<0.01, \mathrm{ON}-\mathrm{G}$ vs $\mathrm{ON}-\mathrm{NB} \mathrm{p}=0.007)$. All CR and $\mathrm{ON}$ mice had smaller liver than corresponding control mice (Supplementary Fig. S1). However, there was no impact of the type of bedding or lack of bedding on the liver size in relation to their body size in ON as well as CR groups. Similarly, CR mice had less epididymal white adipose tissue (eWAT) (Supplementary Fig. S1) and subcutaneous white adipose tissue (sWAT) (Supplementary Fig. S1) than control groups, however, there was no impact of the bedding type. Despite comparable total body weight loss between the different ON groups, the ON-NB group showed higher sWAT content compared to the ON-W, $\mathrm{ON}-\mathrm{C}$, and $\mathrm{ON}-\mathrm{CC}$ groups.

Cage bedding impacts ghrelin and glucose levels in plasma. Mice from CR and ON groups showed increased plasma total ghrelin levels compared to ad libitum groups (Fig. 1e; all groups $\mathrm{p}<0.005$ ). CR-W had a lower level of total ghrelin compared to CR-CC, however, the difference was not statistically significant $(p=0.06)$. The more pronounced pattern was measured for active ghrelin with values for $\mathrm{CR}-\mathrm{W}$ stronger contrasting other $\mathrm{CR}$ groups and showing significant difference when compared to $\mathrm{CR}-\mathrm{C}(\mathrm{p}=0.004$; Fig. 1f). However, each day of $\mathrm{CR}$, mice from all CR groups took a similar time to reach for a daily food portion suggesting equal hunger level (Supplementary Fig. S2). Additionally, the expression of Neuropeptide Y (Npy), leptin receptor (Lepr), and cholecystokinin receptor (Cckr) genes is modified by CR and ON fasting but not affected by the type of bedding (Supplementary Fig. S2). Concerning the ON groups, the type of bedding or lack thereof did not influence plasma active ghrelin levels (Fig. 1f).

$\mathrm{CR}$ and ON groups had generally lower plasma glucose levels compared to ad libitum fed groups (Supplementary Fig. S2). Glucose concentration was lower in ON-NB and ON-G than other ON groups; however, due to multiple groups, not statistically significant in all instances ( $\mathrm{ON}-\mathrm{W}$ vs $\mathrm{ON}-\mathrm{NB} p=0.008$, ON-C vs ON-NB $p=0.03$, $\mathrm{ON}-\mathrm{CC}$ vs ON-NB p $=0.05$, ON-W vs ON-G p $=0.01$, ON-C vs ON-G p =0.04, ON-CC vs ON-G p = 0.03). Upon glucose load mice from ON-NB and ON-G groups showed decreased blood glucose levels starting from $30 \mathrm{~min}$ after glucose administration (Fig. 1g, Supplementary Fig. S2) suggesting increased uptake in peripheral tissues. The area under the curve of plasma glucose levels was smaller for ON-NB and ON-G compared to other ON groups $(\mathrm{ON}-\mathrm{W}$ vs $\mathrm{ON}-\mathrm{NB} \mathrm{p}=0.004, \mathrm{ON}-\mathrm{C}$ vs $\mathrm{ON}-\mathrm{NB} \mathrm{p}=0.008$, ON-CC vs ON-NB $\mathrm{p}=0.003$, ON-W vs ON-G $\mathrm{p}=0.009$, ON-C vs ON-G p =0.01, ON-CC vs ON-G p =0.004; Supplementary Fig. S2). 
a Bedding consumed
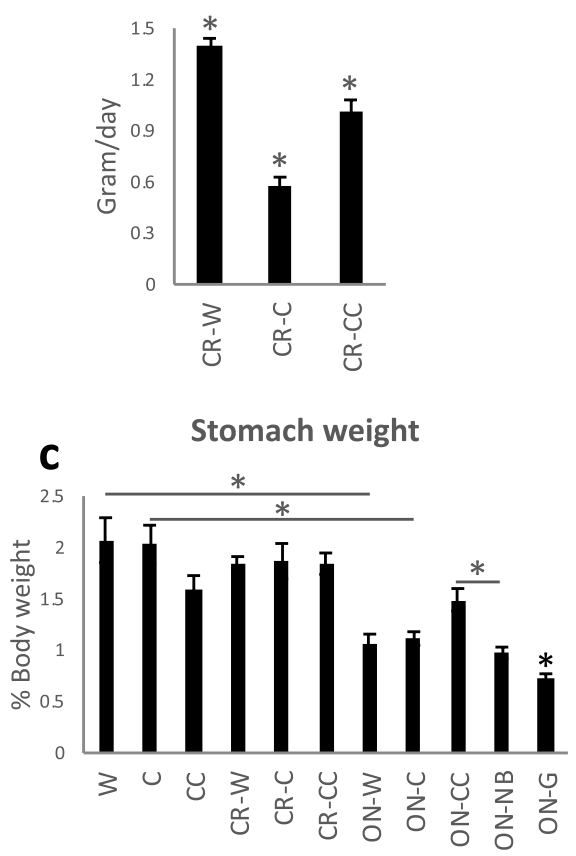

e

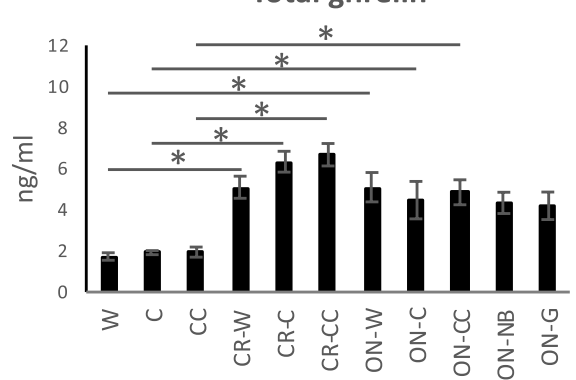

b

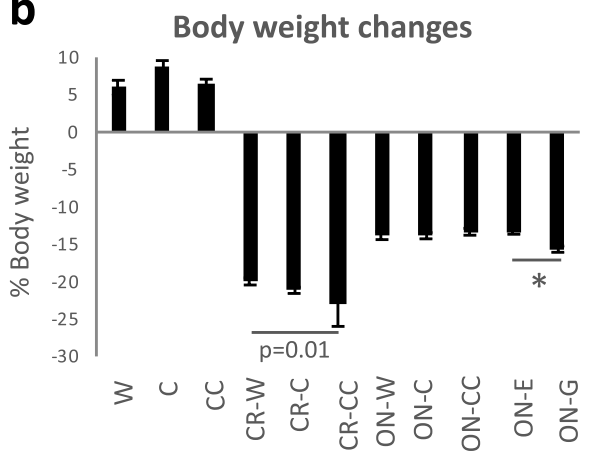

d Cecum weight

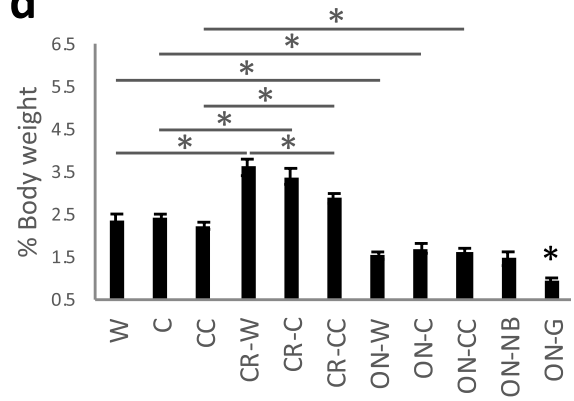

f

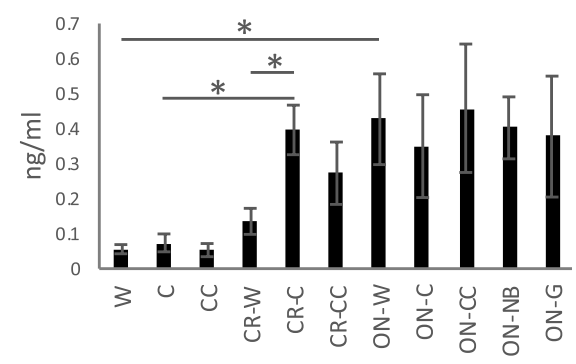

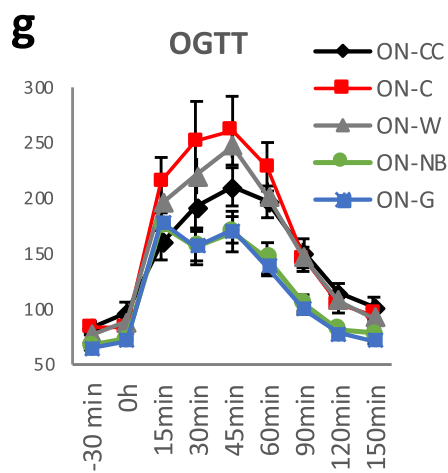

$\mathbf{h}_{\text {Daily feces production }} \mathbf{i} \quad$ Fecal energy content
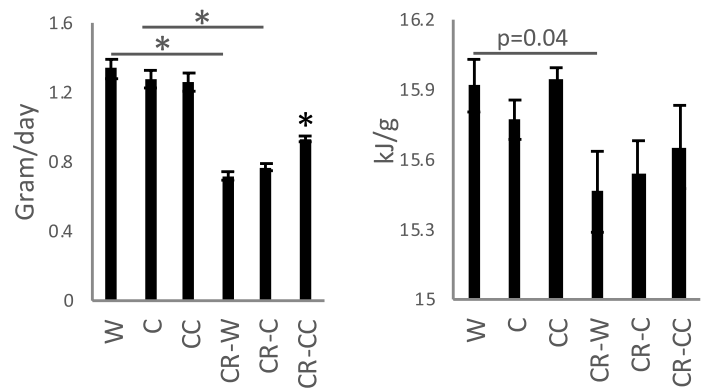

Figure 1. Cage bedding affects body parameters in dietary restricted mice. The amount of bedding consumed by mice submitted caloric restriction (CR) was measured daily between days 11 and 13 of the CR (a). Bodyweight changes were recorded for ad libitum, CR mice as well as over-night (ON) fasted mice and expressed as \% change (b). Stomach (c) and cecum (d) weight were measured. Total (e) and active (f) ghrelin concentrations were analyzed in the mice plasma. Oral glucose tolerance test (OGTT) was performed after ON fasting (g). CR mice feces were collected and its weight (h), as well as energy content (i), was assessed. One-way ANOVA was applied to verify statistical significance. Asterisk $\left({ }^{*}\right)$ indicates statistical significance between the indicated groups after Bonferroni correction for multiple testing. The bars indicate the mean of eight to ten biological replicates \pm SEM. 
Cage bedding affects energy content, microbiota, and metabolites in CR mice cecum. To investigate the impact on the gastrointestinal tract we measured gene expression in the intestinal mucosa. As we previously published ${ }^{5}$, CR tends to increase the expression of metabolic genes (Ppara $\left.\alpha, A c o t 4, S c d 1\right)$ and decrease the expression of inflammatory genes (MyD88, Rsad2, Oasl1a, Irf7) (Supplementary Fig. S3). However, the type of bedding did not affect gene expression.

The bedding was collected from CR mice cages every $24 \mathrm{~h}$ for three consecutive days and feces were separated, dried, and weighed. The CR mice produced fewer feces than ad libitum groups (for all groups $\mathrm{p}<0.001$; Fig. 1h) and the feces contained less energy; however the difference was not statistically significant when correcting for the number of experimental groups ( $\mathrm{W}$ vs CR-W $\mathrm{p}=0.04$; Fig. 1i). Importantly, CR-CC produced more feces compared to the other CR groups (for both groups comparison $\mathrm{p}<0.001$; Fig. 1h). Further, we analyzed cecal microbiota as fermentation of indigestible food in mice is compartmentalized in the cecum. At the same time, microbiota and metabolites share similarities between cecum, colon, and feces ${ }^{20,21}$. All ad libitum fed mice groups shared similar microbial composition (Fig. 2a). We observed a strong shift in cecal bacteria composition from ad libitum to CR mice (Fig. 2a). In general, among the CR groups, CR-W and CR-C bacteria composition overlapped while CR-C was distinct (Fig. 2b-c). CR was accompanied by a non-statistically significant shift in the ratio of Firmicutes to Bacteroidetes (CC vs CR-CC p $=0.01$; Supplementary Fig. S4). The strongest differences on the phylum level were recorded for Proteobacteria (all ad libitum vs CR groups $p<0.001$; Fig. 2 d, Supplementary Fig. S4) and Deferribacteres (all ad libitum vs CR groups $\mathrm{p}<0.001$; Supplementary Fig. S4). The sequencing results were compared to published CR reports ${ }^{22-26}$ and the previously observed decrease in abundance of Roseburia (C vs CR-C p =0.004, CC vs CR-CC p <0.055), Butyricicoccus (all ad libitum vs CR groups $\mathrm{p}<0.001$ ), Streptococcus (W vs CR-W p $=0.005$, C vs CR-C p $<0.001$, CC vs CR-CC $\mathrm{p}<0.001$ ), Anaerotruncus (C vs CR-C $\mathrm{p}=0.001$ ), and Lachnospiraceae (all ad libitum vs CR groups $\mathrm{p}<0.001$; Supplementary Fig. S4) as well as an increase in Lactobacillus (all ad libitum vs CR groups $\mathrm{p}<0.001$ ), Parabacteroides ( $\mathrm{W}$ vs CR-W $\mathrm{p}=0.02$, $\mathrm{C}$ vs CR-C $\mathrm{p}<0.001$, CC vs CR-CC $\mathrm{p}<0.01$ ), and Odoribacter (W vs CR-W p $=0.02$, C vs CR-C $\mathrm{p}=0.03$, CC vs CR-CC $\mathrm{p}<0.001$ ) in CR compared to ad libitum (Fig. 2e,f, Supplementary Fig. S4) were observed. In other reported CR-affected Operational Taxonomic Units (OTU) (e.g. Alistipes, Alloprevotella, Erysipelotrichaceae, Intestinimonas, Lachnoclostridium, Marvinbryantia, Roseburia, Ruminococcaceae ${ }^{23-26}$ we observed inverse or no effect of CR (Supplementary Fig. S5). Importantly, there has also been little overlap between different published data sets. We identified phylum Deferribacteres (CR-W vs CR-CC $\mathrm{p}<0.001, \mathrm{CR}-\mathrm{C}$ vs CR-CC $\mathrm{p}=0.003$ ), genus Lactobacillus (CR-W vs CR-CC $\mathrm{p}<0.006, \mathrm{CR}-\mathrm{C}$ vs CR-CC $\mathrm{p}=0.03$ ) as well as the families Marinifilaceae (CR-W vs CR-CC $\mathrm{p}<0.001, \mathrm{CR}-\mathrm{C}$ vs CR-CC $\mathrm{p}<0.001$ ), Clostridiales XIII UCG-001 (CR-W vs CR-CC $\mathrm{p}<0.001$, CR-C vs CR-CC p $<0.001$ ), Deferribacteraceae (CR-W vs CR-CC $\mathrm{p}<0.001$, CR-C vs CR-CC $\mathrm{p}=0.008$ ), Burkholderiaceae (CR-W vs CR-CC $\mathrm{p}=0.009$, CR-C vs CR-CC $\mathrm{p}<0.001$ ), and Tannerellaceae (CR-C vs CR-CC $\mathrm{p}=0.008$ ) as bacteria affected the strongest (based on $p$-value) by the bedding type in CR mice, particularly by the difference between $\mathrm{CC}$ versus $\mathrm{C}$ and $\mathrm{W}$ beddings (Fig. 2e,g-k, Supplementary Fig. S4). Moreover, OTUs Odoribacter (CR-W vs CR-CC $\mathrm{p}<0.001$, CR-C vs CR-CC $\mathrm{p}<0.001$ ), Mucispirillum (phylum Deferribacteres) (CR-W vs CR-CC $\mathrm{p}<0.001$, CR-C vs CR-CC $\mathrm{p}=0.003$ ), Parasutterella (CR-W vs CR-CC $\mathrm{p}=0.02$, CR-C vs CR-CC $\mathrm{p}=0.002$ ), and Erysipelatoclostridium (CR-W vs CR-CC p <0.001, CR-C vs CR-CC p =0.007), Eubacterium (from Xylanophilum group; CR-W vs CR-CC $\mathrm{p}=0.019$, CR-C vs CR-CC $\mathrm{p}=0.007$ ), Ruminoclostridium 6 (CR-W vs CR-CC $\mathrm{p}=0.03$, CR-C vs CR-CC $\mathrm{p}=0.002)$ and Ruminococcus 1 (CR-W vs CR-CC p $=0.05$, CR-C vs CR-CC $\mathrm{p}=0.001$ ) distinguished CR-CC from CR-W and CR-C (Supplementary Figs. S4 and S6). Correspondingly, the composition of cecal metabolites was affected by the restriction and bedding type (Fig. 3a). The metabolites composition was similar in the mice groups fed ad libitum while among CR groups, CR-W and CR-C were distinct from CR-CC. Variable importance for prediction (VIP) scores were calculated from the PLS and the top 25 highly significant metabolites that cause the difference between the groups have been identified (Fig. 3b). The hierarchical clustering of metabolites further visualized the differences between the groups (Fig. 3c). Among the metabolites setting the CR groups apart, fumaric acid (CR-W vs CR-CC $p=0.03$, CR-C vs CR-CC $p=0.002)$, fructose (CR-W vs CR-CC $\mathrm{p}=0.001$, CR-C vs CR-CC $\mathrm{p}=0.001$ ), and phosphoric acid monomethyl ester (CR-W vs CR-CC $\mathrm{p}=0.02$, CR-C vs CR-CC $\mathrm{p}=0.001$ ) were identified (Fig. $3 \mathrm{c}-\mathrm{f}$ ). The levels of SCFAs acetate (CR-W vs CR-CC $\mathrm{p}=0.01$, CR-C vs CR-CC $p=0.006)$ and butyrate $(C R-W$ vs CR-CC $p=0.001$, CR-C vs CR-CC $p=0.002)$, as well as medium-chain fatty acids (MCFAs), were decreased in all CR groups regardless of the type of bedding (Fig. 3g-i, Supplementary Fig. S7). The levels of propionate were significantly downregulated only for the CR-CC group (CC vs CR-CC $\mathrm{p}=0.001, \mathrm{CR}-\mathrm{W}$ vs CR-CC $\mathrm{p}=0.001, \mathrm{CR}-\mathrm{C}$ vs $\mathrm{CR}-\mathrm{CC} \mathrm{p}=0.001$; Fig. 3i). Further, we sought to identify which bacteria could contribute to the observed metabolomic changes. A correlation between changes in bacteria composition and metabolites occurrence revealed the co-dependence of multiple factors (Fig. 4, Table 1). Correlation coefficients were calculated for the CR groups and depicted by Cytoscape into clusterings of cecal bacterial families and metabolites indicating strong interaction (Fig. 4).

\section{Discussion}

Previously we showed that mice submitted to CR increased their cecum weight as a likely result of the accumulation of indigestible fiber from bedding ${ }^{5}$. In the current project, we investigated the consequences of the increased consumption of cage bedding and how it influences the outcome of animal studies. We show the impact of cage bedding in restrictive dietary protocols, reflected by differences in body weight, adiposity, cecum size, glucose, and ghrelin levels in plasma as well as cecal microbiota and metabolites profile.

All CR mice consumed bedding, however, the amounts of eaten beddings varied suggesting a difference in preference (olfactory and gustatory properties), the capacity of the bedding to deliver energy, or offer satiety. The CR groups showed bedding-dependent variability in weight loss. The difference may be explained by the availability of fiber which, especially the soluble one, can serve as a source of energy $(2 \mathrm{kcal} / 100 \mathrm{~g})^{27}$. CR-W and CR-C 


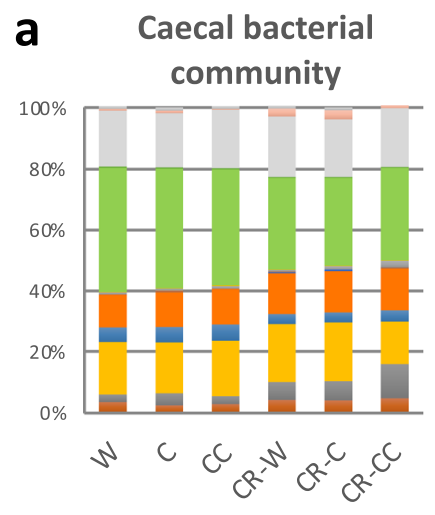

f Akkermansiaceae f_Erysipelotrichaceae f_Rumino coccaceae - f Peptococcaceae f_Lach nospiraceae - $f$ Clostridiales Family XIII f_Clostridial es vadinBB60 a_Christensenellaceae f_Tannerellaceae f_Riken ellaceae - f_Prevotellaceae f_Muribaculaceae - $\mathrm{f}$ Marinifilaceae - f_Bacteroidaceae - $f_{\text {_Eggerthellaceae }}$ b Microbial diversity in the caecum

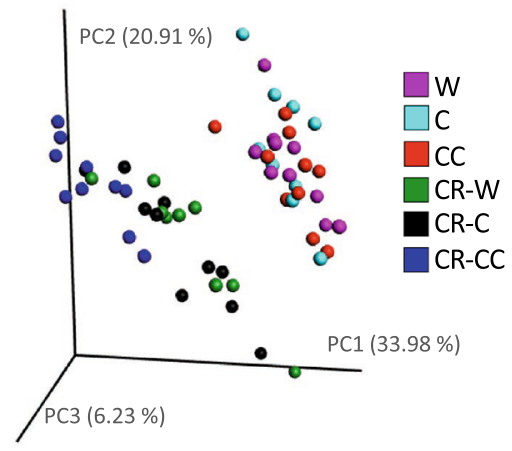

\section{C}

Hierarchical clustering of bacterial families

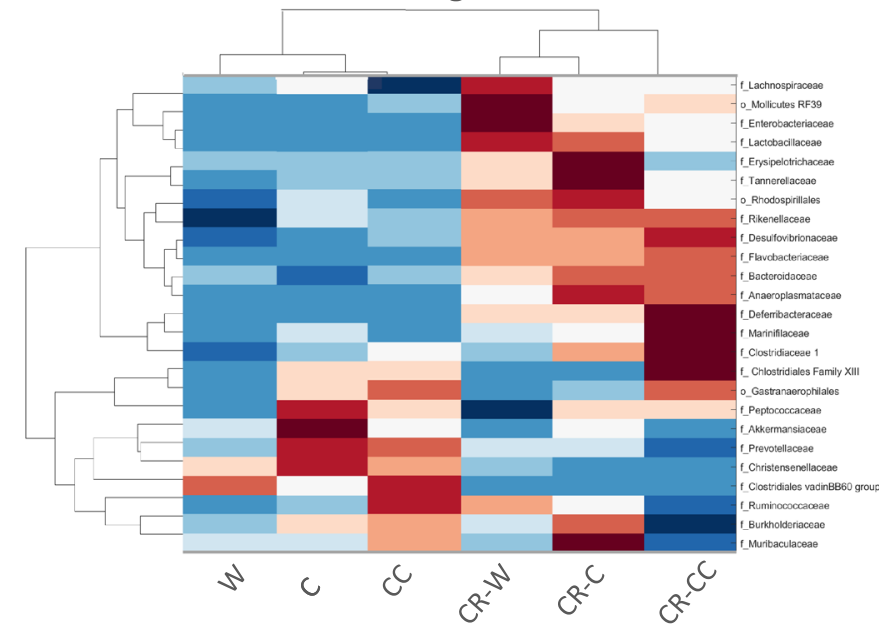

d Proteobacteria

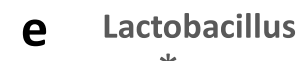

f Parabacteroides

g Marinifilaceae

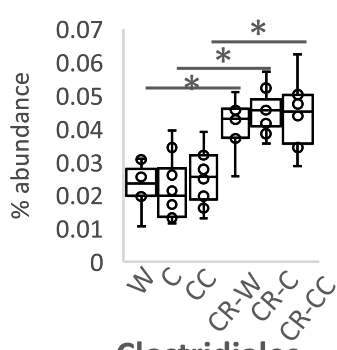

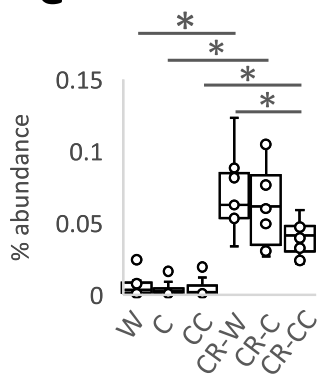

\section{i}

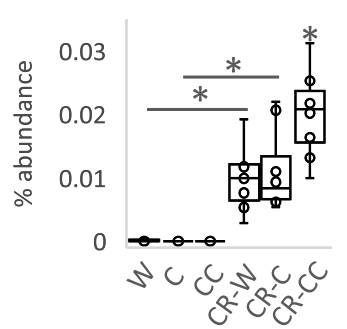

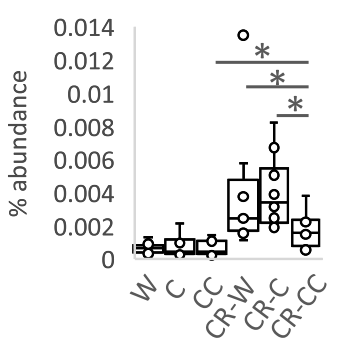

Burkholderiaceae

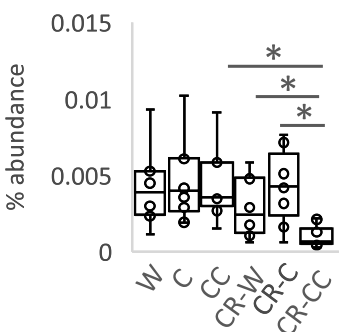

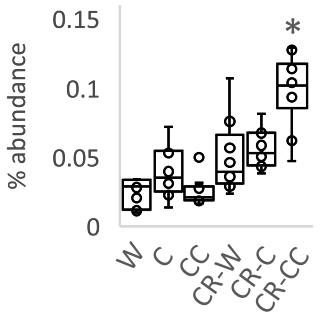

k Tannerellaceae

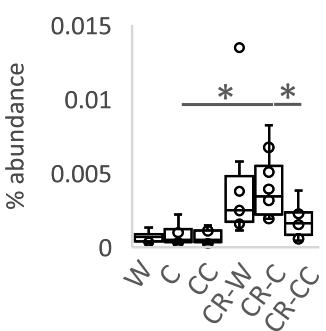

Figure 2. Cage bedding impacts the composition of cecal microbiota. The composition of bacterial families (a) and microbial diversity (b) in the cecum was analyzed in ad libitum and CR fed mice. The data was presented as a heatmap of the hierarchical clustering analysis of bacterial families using COVAIN (c). The abundance of bacteria in the cecum was expressed as $\%(\mathbf{d}-\mathbf{k})$. Asterisk $\left(^{\star}\right)$ indicates statistical significance after Bonferroni correction for multiple testing. Groups were compared using one-way ANOVA. Error bars stand for the mean \pm SEM. The data represents nine to ten biological replicates per experimental group. 
a Caecal metabolic profile

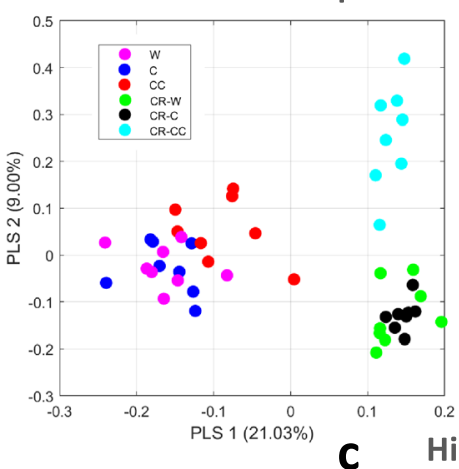

b PLS VIP score

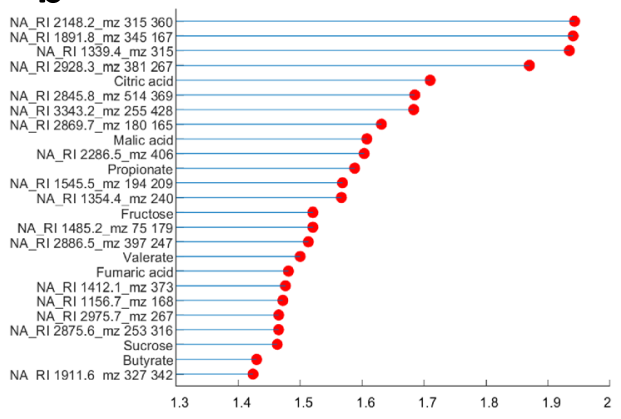

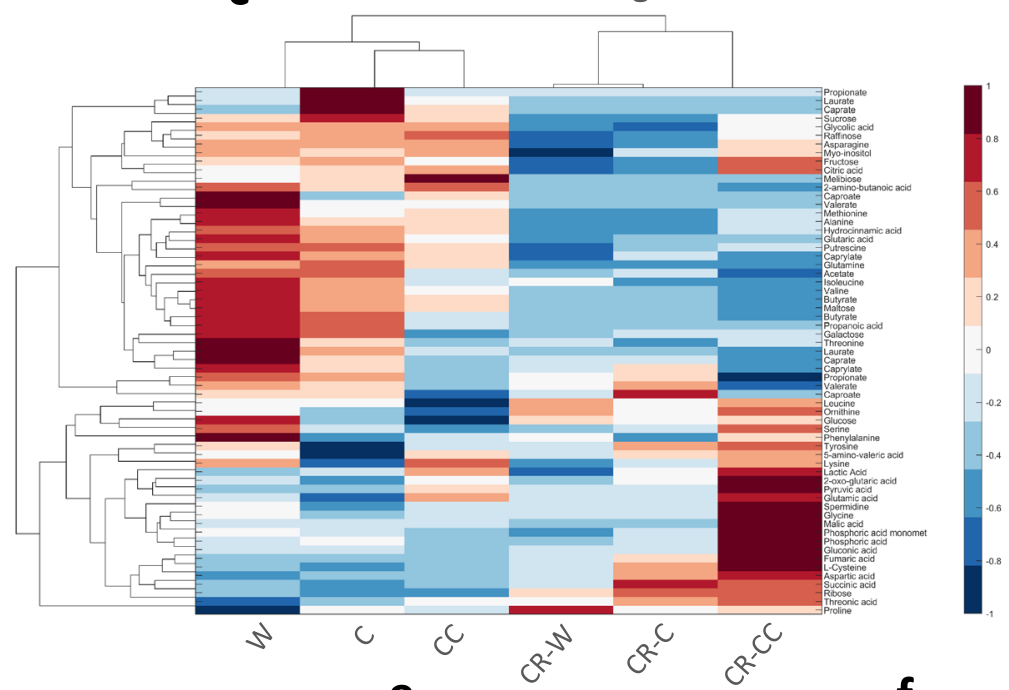

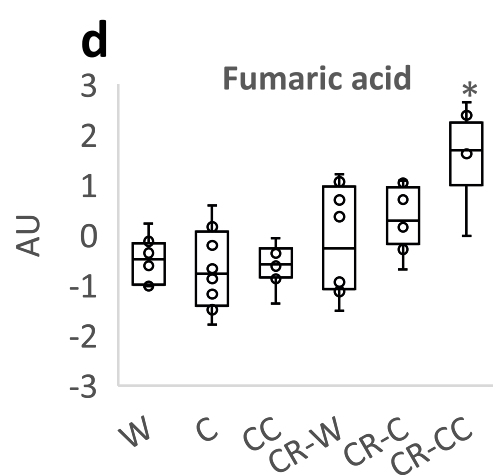

g

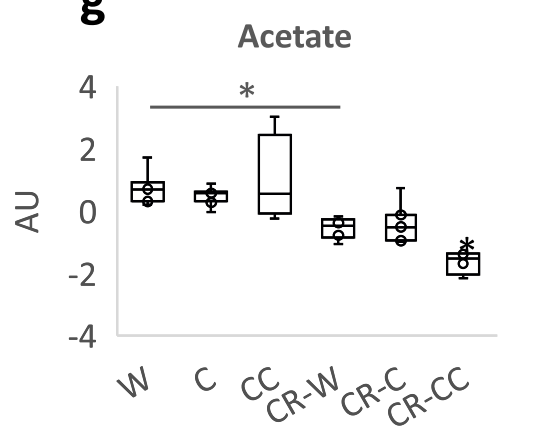

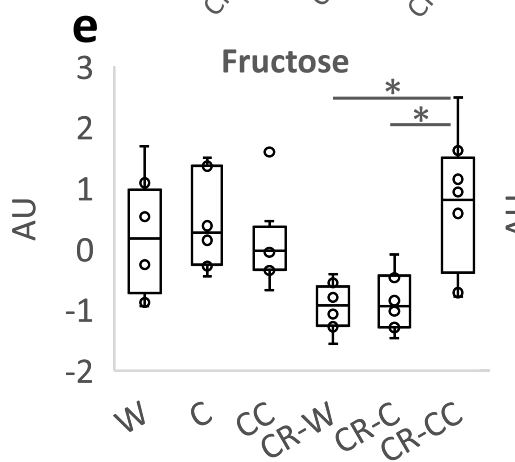

h

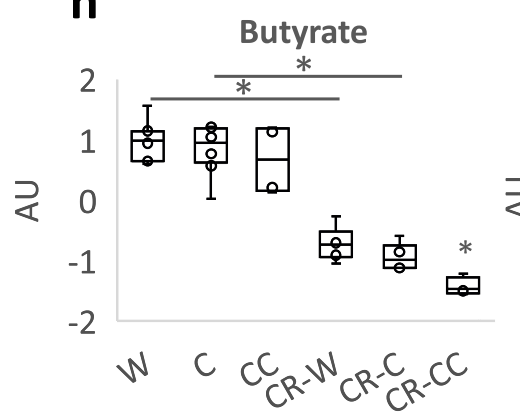

Phosphoric acid

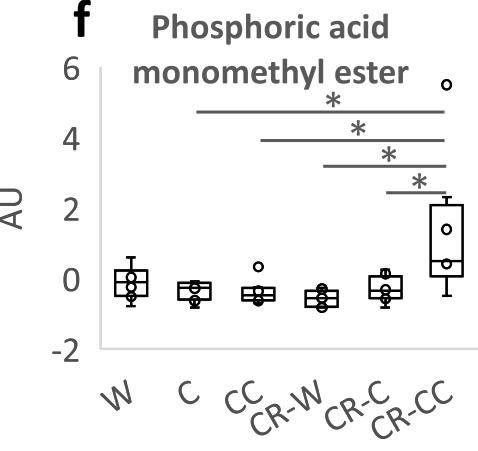

Propionate

3

2

1

$-1$

$-3$

W $C C_{C R} C-W_{C R}-C_{C R}-C$

Figure 3. Cage bedding impacts the composition of cecal metabolites. The metabolome of cecal content was analyzed (a) and the most important variables were summarized (b). Heatmap of hierarchical clustering analysis of annotated metabolites was created using COVAIN (c). Z-Scored metabolites figures show the relative deviation from the groups mean value (0) for fumaric acid (d), fructose (e), and phosphoric acid monomethyl ester (f) represent the most important annotated metabolites contributing to a distinct metabolic profile within the CR group. The cecum content of SCFAs was analyzed $(\mathbf{g}-\mathbf{i})$. Single data points are indicated by circles and medians as horizontal lines within each box. One-way ANOVA was applied to verify statistical significance. Asterisk $\left.{ }^{*}\right)$ indicates statistical significance after Bonferroni posthoc analysis. Error bars stand for $\pm S E M ; n=8-10$. 


\section{Network of bacteria and metabolites}

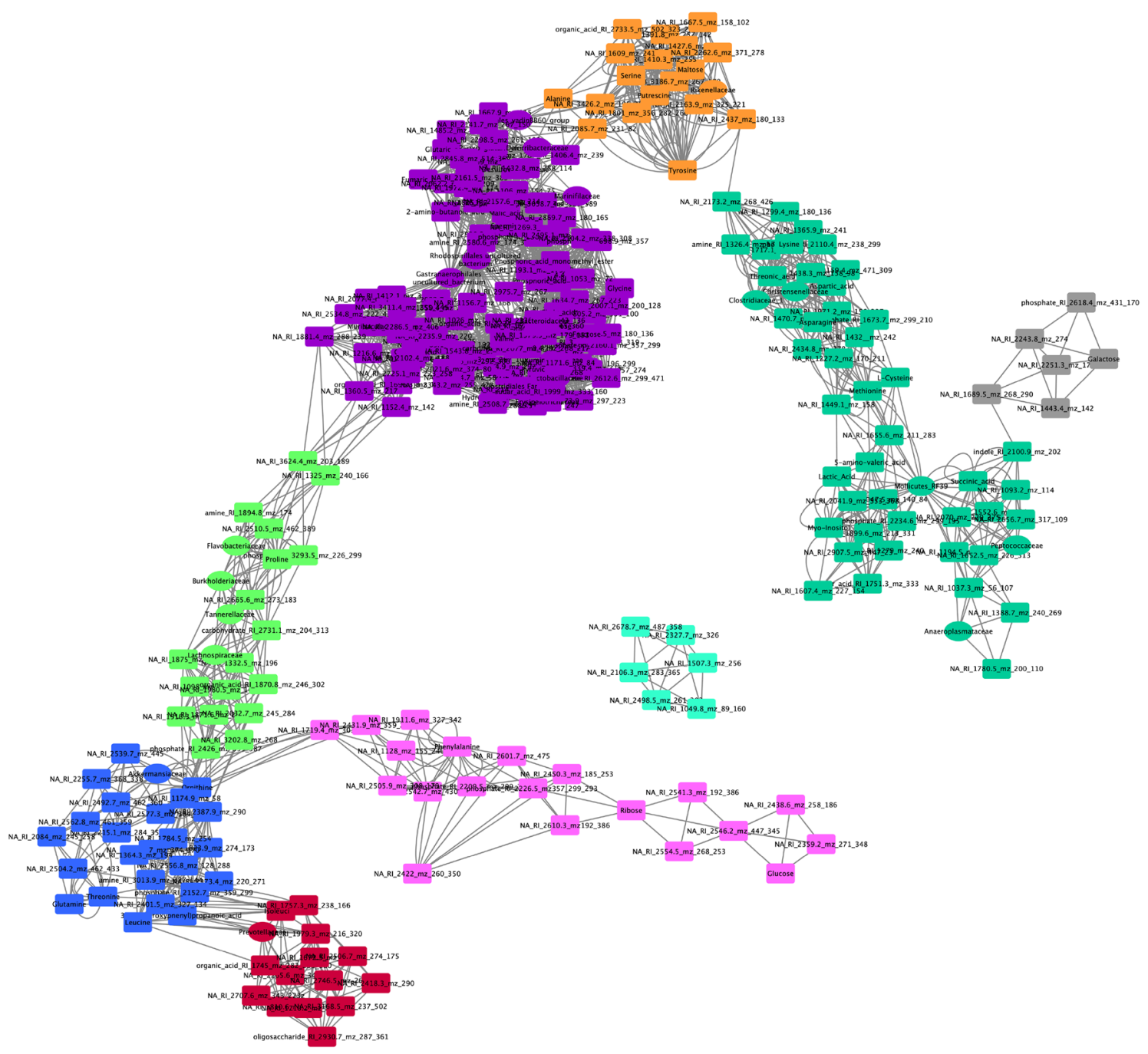

Figure 4. Correlation network of bacteria with metabolites in the cecum. The correlation network depicts changes in bacterial families composition and metabolites occurrence characteristic to CR. Each node represents one metabolite (ellipse) or a bacterial family-level OUT (V-shape) and each edge represents a statistically significant correlation where the Pearson's correlation coefficient $\geq 0.8$. Girven-Newman algorithm was applied in network clustering analysis where modules (clusters, denoted by different colors) depict association patterns between metabolites and bacteria. The visualization was performed with Cytoscape v3.7.2. (http://www.cytos cape.org/).

groups consumed mostly cellulose which is fermentable to some extent (up to 70\%-80\%), even in humans ${ }^{28,29}$. Similarly, hemicellulose can be bacterially digested ${ }^{29}$ and it might improve the fermentability of cellulose $e^{30}$. While lignin is indigestible by human enzymes ${ }^{29}$, it was shown in vitro to be partially digestible by the human microbiome ${ }^{31}$ and in vivo by rats ${ }^{32}$. In order to compare the amount of energy taken up and extracted by mice from the ingested beddings energy content in feces was measured. We were not able to detect differences in fecal energy content $(\mathrm{kJ} / \mathrm{g})$ between the CR groups, however, it is important to notice that the CR-CC group produced more feces (g/day) and therefore secreted more total energy ( $\mathrm{kJ} /$ day). This result is accompanied by the highest body weight loss for the CR-CC group. Therefore, we suspect that of the three beddings CC was the source of which the mice were able to absorb the least of energy. Importantly, it is impossible to state whether the differences in the measured amounts of feces result from disparities in the production or the consumption of feces.

The weight of the stomach with its content was considered in our study as an indicator of the scale of consumption within the last hours prior to the dissection. The smaller stomach size in the animals housed on the grids proved our concept. Interestingly, although ON fasted animals consumed bedding their stomachs were much smaller than the stomachs of CR mice, whereas the difference between ad libitum and CR mice was minor. Therefore, prolonged restriction favors enhanced bedding consumption. Similarly to the stomach, the weight of the cecum with its content reflected the amount of fiber consumption. However, this was more specific in a way that it also pictured the intake over a prolonged period, fiber accumulation, and digestibility of the fiber in 


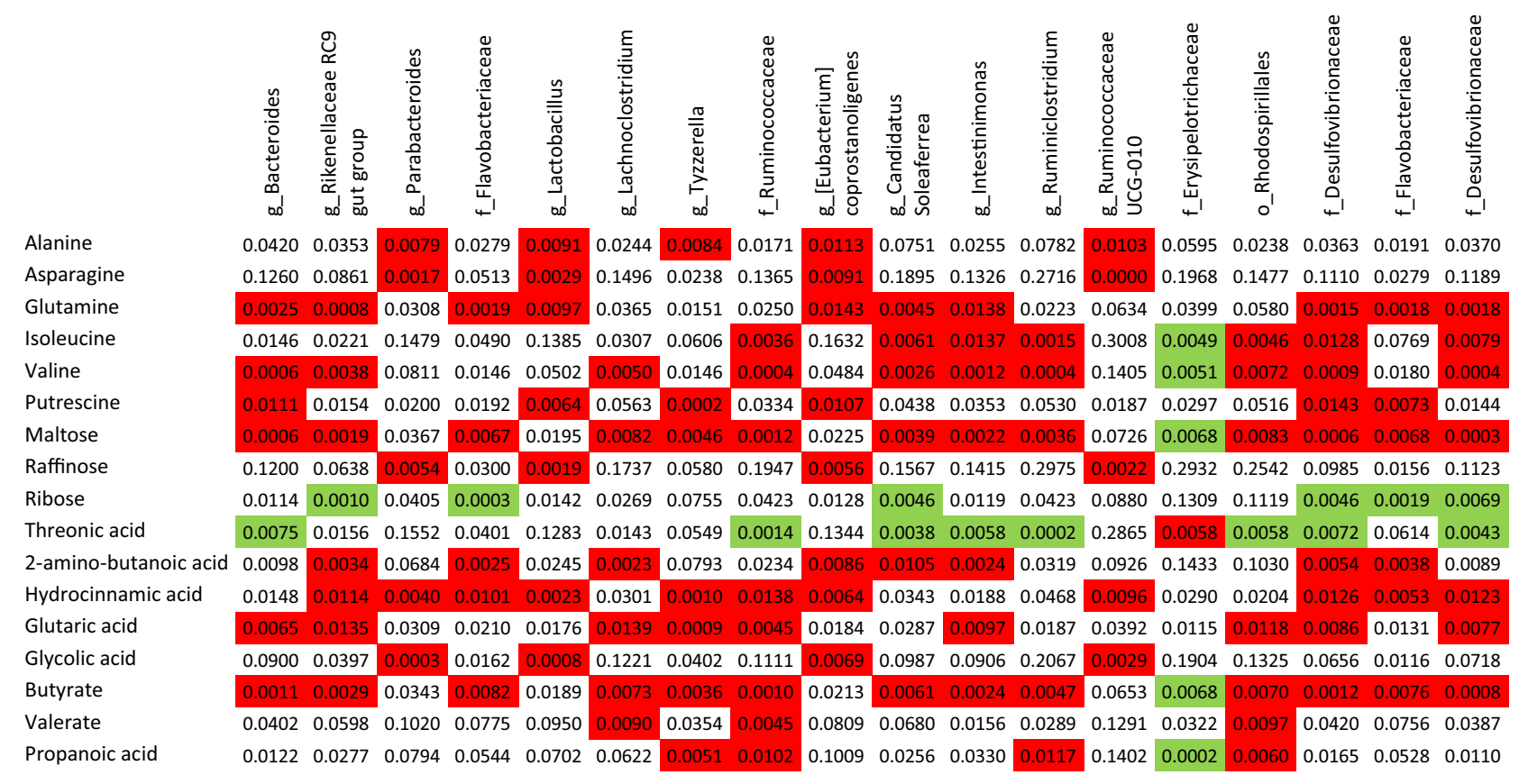

Table 1. Correlation of bacteria with metabolites in the cecum. Correlation p-values between annotated metabolites and the bacterial genus in the cecum of CR mice were calculated. For Operational Taxonomic Units (OTU) that could not be assigned on the genus level, the closest taxonomical level of identification was used. Characters before the name of the bacteria represent family (f), genus (g), and order (o). Coloured cells show statistically significant differences; red = negative correlation; green $=$ positive correlation. Correlation coefficient analysis using Pearson's correlation $(\mathrm{r}=0.8)$ was done in COVAIN; $\mathrm{p}<0.0083$.

the given beddings. Interestingly, the size of the cecum reversely correlated with the amount of feces produced as CR mice had a smaller cecum when housed on CC bedding.

Since bedding and feces ingestion influence the outcomes of metabolic studies ${ }^{14,33,34}$ rodents may be kept on a metal grid short-term ${ }^{35}$ to prevent bedding consumption as well as limit coprophagy. However, this experimental setup cannot stop mice from eating feces directly from the anus. To completely cease coprophagy, the use of anal cups or neck restrainers would be necessary, which would be distressing to the animal and could interfere with normal feeding behavior. In our study, mice housed on the grid showed higher body weight loss as well as lighter stomach and cecum compared to conventionally fasted mice, indicating less consumption. It has previously been shown that rats housed in wire-bottom cages do not show any clinical pathology symptoms when compared to rats from solid bottom cages $^{36}$. However, housing rats in wire-bottom cages overnight leads to immediate alterations of heart rate, body weight, and locomotor activity, which might be related to stress response ${ }^{37}$. Therefore, the bodyweight loss in the mice fasted on the grid may also indicate the impact of additional stress or energy loss required for body temperature regulation when deprived of nesting material. When coprophagy was permitted, ON fasted mice did not differ in cecum and body weight from mice with access to bedding indicating the importance and scale of coprophagy. Coprophagy occurs not only to compensate for energy during deprivation but also to supplement the diet with various nutrients of gut microbiota origin. Choline, cysteine, thiamine, iron, vitamin $\mathrm{K}, \mathrm{B}_{12}$, and essential fatty acids are sourced from feces and if the animals are not allowed to consume feces, chow needs to be supplemented ${ }^{38-46}$. However, on an ad libitum balanced laboratory diet coprophagy might not be crucial, as its absence does not result in lower body weight or less progeny ${ }^{47}$. Most importantly, coprophagy leads to the re-inoculation of the gut, therefore, it is an important factor in microbiota composition. Moreover, bedding presence and not coprophagy was the deciding factor concerning glucose tolerance. Both groups, housed without bedding or kept on the grid showed lower glucose levels compared to any group submitted to ON in the presence of bedding despite the fact that the initial, fasting glucose levels were comparable between all the groups.

Since CR results in hunger, the impact of supplementation with different beddings on hunger was tested by assessing the speed of meal initiation. Bedding did not influence how rapidly the mice started consumption of the daily portion of chow. Nevertheless, after day two of CR, all groups ate nearly immediately following access to food, therefore, beyond this point small differences in the speed of the meal initiation were difficult to detect. Moreover, basal blood glucose levels and gene expression in the hypothalamus were comparable between the animals of different CR and ON groups. Importantly, the levels of active ghrelin, the "hunger hormone", were increased in CR-CC and CR-C compared to CR-W. This indicates that the type of bedding may influence hunger perception.

Several publications reported changes in microbiota composition in CR compared to ad libitum fed mice. Data comparison revealed a few similarities of our results with previous studies ${ }^{22-26}$ concerning consistent trends in the abundance of selected OTUs, regardless of the type of bedding. Nevertheless, numerous differences in bacteria occurrence were found between ours and the published sequencing results. Obviously, microbiota 
composition depends on multiple factors including different types of cage bedding, contributing to the differences in outcomes of similar experiments. Accordingly, strong differences in gut bacteria and metabolites were observed between beddings having the most distinct fiber profile ( $\mathrm{CC}$ vs $\mathrm{C}$ and $\mathrm{W}$ ), while $\mathrm{C}$ and $\mathrm{W}$ bedding, which stem from the same initial material, resulted in comparable microbiota composition. Fittingly, Ruminococcus, which abundance raises in response to high-cellulose diet $^{48}$, was increased in CR-C and CR-W versus corresponding ad libitum groups but this was not present in CR-CC. Consequently, Ruminococcus was identified as one of the OTUs distinguishing CR-CC versus both CR-W and CR-C. Therefore, it is important to consider that the main trigger of microbiota composition changes in CR animal trials is not the nutrient restriction but the bedding consumption.

Lactobacillus which is known to mitigate inflammation and improve gut barrier function is stimulated by $\mathrm{CR}^{22,49}$. Also in our study CR strongly increased the abundance of Lactobacillus in mice cecum. This result corresponds well with our previous report ${ }^{5}$ showing a decrease in expression of the immune function-related gene in the intestine of CR mice. Importantly, the type of bedding affected the extent to which the bacteria levels increased, therefore, likely modulating inflammatory status.

A high-fiber diet affects body weight, GI-tract and liver status, microbiota composition, fiber fermentation, and general health ${ }^{8,50,51}$. However, most of the studies point toward a stronger impact of soluble versus insoluble fiber since these are the vital substrates for SCFA production ${ }^{16,50,52,53}$. Despite high fiber intake and cecum enlargement suggesting an elevated fermentation, CR mice showed lowered levels of all short to medium-chain fatty acids. The lowered levels of SCFA may be associated with the decrease in the relative abundance of enzymes involved in butyrogenesis and acetogenesis upon $\mathrm{CR}^{23}$. However, an increase in propiogenesis-related enzymes has been reported in parallel ${ }^{23}$. Moreover, supplementation with fiber including cellulose or corn fiber does not result in an increased SCFA in the cecum ${ }^{54,55}$. The knowledge concerning insoluble fiber fermentation by gut microbiota is very limited. In general, compared to soluble fiber, cellulose is poorly fermented, it yields mostly acetate and less propionate or butyrate ${ }^{54,55}$, and the process takes place mostly in the distal colon where transit time is slower, and bacterial densities are higher ${ }^{56}$. There are multiple fecal strains digesting cellulose from both major phyla Bacterioidetes and Firmicutes ${ }^{57}$ and the main cellulolytic strains isolated from human feces have been classified as Ruminococcus sp, Clostridium sp, Eubacterium sp, and Bacteroides sp. ${ }^{57-60}$. Therefore, despite the lower fermentability of cellulose compared to soluble fiber, we assume that SCFA production increased in CR mice supplementing themselves with cage bedding. We propose that the produced metabolites are likely taken up more efficiently and utilized by the host due to CR-related energy shortage. Additionally, the levels of fumaric acid, an intermediate product of bacterial fermentation are increased in all CR groups and highest in CR-CC. This implies an increased fermentation particularly strongly stimulated by CC bedding.

We analyzed the correlation between the occurrence of the detected cecal microbiota and corresponding metabolites. We could confirm the previously published negative correlation between Bacteroides and fatty acids $^{61,62}$, Oscillibacter and isoleucine ${ }^{63}$ and positive correlation between Alistipes and proline ${ }^{62}$, Lachnoclostridum and glutaric acid ${ }^{64}$, Bacteroides and spermidine ${ }^{65}$ as well as Blautia and malic acid and myo-inositol ${ }^{62}$. However, most of the observed correlations have not been reported before.

To summarize, based on the differences between mice kept with or without cage bedding we can conclude that mice consume bedding and feces during dietary restrictions and this influences their body, WAT, stomach and cecum weight, cecal microbiota and metabolites profile as well as plasma glucose and ghrelin level. Therefore, we propose that the amount of energy extracted from fiber depends on the bedding type and contributes to the bodyweight differences. Moreover, in the to-date published reports on the effect of CR, particularly on microbiota, in mice or rats, it is impossible to distinguish between the effect of CR or the supplemented bedding making the results not comparable to human CR studies. The reproducibility of published results is a major issue in the scientific community. Our data indicate an important factor that needs to be taken into account when interpreting and designing experiments, particularly important when restrictive diets are considered. With great progress in the field of microbiota within the last few years and knowing the importance of dietary fiber as a source of prebiotics, it is important to indicate that gut bacteria composition is affected by the type of fiber present in mice cage bedding. We propose that for short-term restrictions, cages with grid floors are used. Due to ethical concerns, this approach cannot be applied to long-term experiments, instead it should be encouraged to routinely report not only the type of the diet but also cage bedding.

\section{Materials and methods}

Animal experiments. Male C57BL/6NRj mice purchased from Janvier Inc. Labs (Le Genest, France) were housed in standard SPF conditions using a Tecniplast IVC system (cage type 2L, blue line). Mice were fed a standard chow (V1535 R/M-H Extrudate; ssniff Spezialdiäten GmbH, Soest, Germany). The animals were divided into control ad libitum, CR, and ON fast groups (Supplementary Table S1). Each of these groups was separated into three subgroups by the bedding type: wooden (Lignocel select), corncob (RehoFix MK 3500), cellulose (Arborcel Performance Small; all beddings from J. Rettenmaier \& Söhne GmbH + Co KG; Vienna, Austria). The fiber composition of the beddings is presented in the Supplementary Fig. S1A. Additionally, two subgroups of the ON fast group were housed without any cage bedding or on a metal grid to prevent contact with cage bottom and coprophagy. The control group mice were kept ad libitum on each of the bedding. Each group of mice contained 10 animals; however, during the dissection some of the tissues were lost resulting in 8-10 replicates for the presented results. Mice from CR groups were submitted to two weeks CR with 75\% of normal food intake. Mice body weight was measured daily during the CR protocol. To estimate the hunger of the CR mice, a daily food pellet was placed in the cage and the time it took the mice to initiate the meal was measured with a stopwatch. Cage bedding was changed daily from the $11^{\text {th }}$ day of CR for three consecutive days to assess the amount of bedding eaten. The harvested bedding was dried and feces were separated. The feces were dried 
and used for verification of daily fecal mass production and energy content measurement by direct calorimetry (IKA-Kalorimeter C2000; IKA-Werke GmbH \& Co. KG; Staufen, Germany). Fresh fecal samples were collected on days 12 and 13 of CR and from ad libitum fed mice. The feces were snap-frozen and stored at $-80^{\circ} \mathrm{C}$.

For the ON fasted groups, food was removed in the evening and the mice were fasted $16 \mathrm{~h}$ with free water access. After ON fast, mice were submitted to an oral glucose tolerance test (OGTT) by gavaging 3 mg glucose per gram body weight. Afterward, the mice were fed ad libitum. One week later the ON fasting procedure was repeated and mice were sacrificed and dissected in the morning. Food from the cages of control mice was removed $2 \mathrm{~h}$ before the dissection. All mice were euthanized by isoflurane overdose, with blood drawn by cardiac puncture. The stomach and cecum with their content as well as adipose tissue and liver weight were recorded. Blood was mixed with $10 \mu \mathrm{l} / \mathrm{ml}$ EDTA, $20 \mu \mathrm{l} / \mathrm{ml}$ aprotinin, and $10 \mu \mathrm{l} / \mathrm{ml}$ dipeptidyl peptidase (DPP) IV. Plasma was separated from the blood cells by centrifugation for $10 \mathrm{~min}$ at $3,600 \mathrm{xg} 4{ }^{\circ} \mathrm{C}$ and was stored at $-80^{\circ} \mathrm{C}$. Stomach, small intestine, and colon scrapings as well as cecum content were snap-frozen and stored at $-80^{\circ} \mathrm{C}$ until use.

All animal experimentation protocols were approved by the Federal Ministry of Science, Research and Economy, Unit for Animal Experiments and Genetic Engineering in Austria (BMWFW-66.006/0017-WF/V/3b/2016). The experiments were performed in agreement with the Austrian Federal Act on Animal Welfare.

Sequencing the 16S rDNA genes and metataxonomic analysis. The samples for sequencing were processed according to the previously published protocol ${ }^{66}$. Cecum samples were homogenized in MagNA Pure Bacteria Lysis Buffer from the MagNA Pure LC DNA Isolation Kit III (Bacteria, Fungi) in MagNA Lyser green beads tubes at 6,500 rpm for three $30 \mathrm{~s}$ cycles in a MagNA Lyser Instrument (all from Roche, Mannheim, Germany). The homogenized samples were mixed with $25 \mu \mathrm{l}$ lysozyme $(100 \mathrm{mg} / \mathrm{ml})$, incubated at $37^{\circ} \mathrm{C}$ for $30 \mathrm{~min}$ followed by adding $43.4 \mu \mathrm{l}$ Proteinase $\mathrm{K}(20 \mathrm{mg} / \mathrm{ml})$ and incubation at $65^{\circ} \mathrm{C}$ overnight. Afterwards, the enzymes were heat-inactivated at $95^{\circ} \mathrm{C}$ for $10 \mathrm{~min}$ and $250 \mu \mathrm{l}$ lysed supernatant was used for DNA extracted on a MagNA Pure LC 2.0 following the instructions for the MagNA Pure LC DNA Isolation Kit III (Bacteria, Fungi) (Roche). PCRs reactions were run in triplicates using a FastStart High Fidelity PCR system and contained $5 \mu$ lof total DNA, $1 \times$ Fast Start High Fidelity Buffer, 1.25 U High Fidelity Enzyme, $200 \mu \mathrm{M}$ dNTPs, $0.4 \mu \mathrm{M}$ primers, and PCR-grade water in $25 \mu \mathrm{l}$ reaction volume (all reagents from Roche, Mannheim, Germany). The following target primers were applied for the amplification of phylogenetic informative hypervariable regions V1-V2: 27F-AGA GTTTGATCCTGGCTCAG and 375R-CTGCTGCCTYCCGTA. The primers were used with Illumina adapters for indexing PCR reaction according to Illumina's $16 \mathrm{~S}$ metagenomic sequencing library preparation guide. The PCR temperature cycles were as follows: initial denaturation at $95^{\circ} \mathrm{C}$ for $3 \mathrm{~min}, 30$ cycles of denaturation at $95^{\circ} \mathrm{C}$ for $45 \mathrm{~s}$, annealing of primers at $55^{\circ} \mathrm{C}$ for $45 \mathrm{~s}$ and extension at $72{ }^{\circ} \mathrm{C}$ for $1 \mathrm{~min}$, final extension step at $72{ }^{\circ} \mathrm{C}$ for $7 \mathrm{~min}$ and cooling to $4^{\circ} \mathrm{C}$. The PCR reaction triplicates were pooled and checked using $1 \%$ agarose gel and subsequent normalization of $20 \mu \mathrm{l}$ PCR products was performed on a SequalPrep Normalization Plate (LifeTechnologies, Germany). Of the normalized PCR products, $15 \mu \mathrm{l}$ was used as a template in a single $50 \mu \mathrm{l}$ indexing PCR reaction for 8 cycles; the temperature cycles conditions were as described above for the targeted PCR. For the final sequencing library, $5 \mu \mathrm{l}$ of PCR products from each sample were pooled and $30 \mu$ of the library was purified using a 1\% agarose gel and the QIAquick gel extraction kit (Qiagen, Germany). The obtained library was quantified with QuantiFluor ONE dsDNA Dye on Quantus Fluorometer (Promega, Germany), its quality was verified using an Agilent BioAnalyzer 2100 (Waldbronn, Germany) and the $6 \mathrm{pM}$ library was sequenced on a MiSeq desktop sequencer (Illumina, Netherlands) containing 20\% PhiX control DNA (Illumina) with v2 chemistry for 500 cycles. FastQ raw reads were used for subsequent data analysis.

Raw sequencing data in fastq format was imported in Galaxy web-based platform ${ }^{67}$ and analyzed with the QIIME2 2018.4 microbiome analysis pipeline. After initial quality control data was preprocessed with DADA2 ${ }^{68}$ using default parameters and removing specific primer sequences. The resulting feature representative sequences were classified with the QIIME2 pre-fitted sklearn-based taxonomy classifier against SILVA 16S rRNA database version 132 at $99 \%$ identity ${ }^{69}$. The resulting feature abundance table, also known as OTUs table over all samples including taxonomy information was used for all subsequent analyses. For the phylogenetic methods, representative sequences were aligned with MAFFT de novo multiple sequence aligner ${ }^{70}$ followed by the creation of a phylogenetic tree with FastTree ${ }^{71}$.

Metabolomics. Extraction and analysis of cecal metabolites were performed according to Weckwerth et al. ${ }^{72}$ with slight modifications. Frozen samples $(\sim 30 \mathrm{mg})$ were transferred into "Precellys lysis kit" homogenizing tubes with $1.4 \mathrm{~mm}$ ceramic beads and $800 \mu \mathrm{l}$ ice-cold MCW extraction buffer (methanol:chloroform:water = 2.5:1:0.5) was added. The samples were homogenized in a Precellys24 Tissue Homogenizer (Bertin Instruments) twice for $15 \mathrm{~s}$ at $5000 \mathrm{rpm}$ and were incubated on ice for $15 \mathrm{~min}$. Next, samples were vortexed and centrifuged for $5 \mathrm{~min}$ at $10,500 \mathrm{rpm}$ at $4{ }^{\circ} \mathrm{C}$ then the supernatant was transferred to a $2 \mathrm{ml}$ Eppendorf tube. The extraction step was repeated by short vortexing the pellet with $400 \mu \mathrm{l}$ ice-cold MCW followed with 15 min incubation on ice and centrifugation for $5 \mathrm{~min}$ at $10,500 \mathrm{rpm}$ at $4{ }^{\circ} \mathrm{C}$. The two supernatants were combined and to separate chloroform phase from the water/methanol phase $400 \mu \mathrm{H} \mathrm{H}_{2} \mathrm{O}$ was added. After vortexing and centrifuging the samples for another $5 \mathrm{~min}$ at $14,000 \mathrm{rpm}$ at $4{ }^{\circ} \mathrm{C}$, the upper polar phase was transferred to a new Eppendorf tube and both fractions were dried in a speed vac using an optimized pressure gradient to prevent boiling retardation. The polar fraction was dissolved in $50 \mu \mathrm{l}$ of methoxamine hydrochloride solution $(20 \mathrm{mg} / \mathrm{ml}$ pyridine $)$ and incubated at $30{ }^{\circ} \mathrm{C}$ for $90 \mathrm{~min}$ with continuous shaking. Then $80 \mathrm{ml}$ of $\mathrm{N}$-methyl- $N$-trimethylsilyltrifluoroacetamid (MSTFA) was added to derivatize polar functional groups at $37^{\circ} \mathrm{C}$ for $30 \mathrm{~min}$. The derivatized samples were stored at room temperature for $120 \mathrm{~min}$ before injection. Gas chromatography-mass spectrometry (GC-MS) analysis was performed using a Leco Pegasus BT-TOF (Leco Instrumente GmbH, Mönchengladbach, Germany) 
equipped with a PAL3 Autosampler (CTC Analytics AG, Zwingen, Switzerland). Chromatographic separation and data validation were conducted as published earlier with slight modifications ${ }^{73,74}$. Derivatized extract $(1 \mu \mathrm{l})$ was injected on an HP-5MS column $(30 \mathrm{~m} \times 0.25 \mathrm{~mm} \times 0.25 \mu \mathrm{m})$ (Agilent Technologies) in split less mode. Mass spectral data acquisition was performed using the following instrument parameters. Electron impact ionization was conducted at $70 \mathrm{eV}$ and $1 \mathrm{~mA}$ emission current. Ion source and transferline temperature were set to $250{ }^{\circ} \mathrm{C}$. Mass spectra were collected at an acquisition rate of 10 spectra/sec and a mass range of 40-600 Th using a relative detector voltage with an offset of $-100 \mathrm{~V}$ from optimized detector voltage. Mass spectrometry data are stored at MetaboLights (https://www.ebi.ac.uk/metabolights/).

Statistical analysis. OTUs table was reduced by removing all OTUs present in less than three samples per group. The obtained data of GC-MS and LC-MS were normalized to fresh weight, then annotated and classified according to the Metabolomics Standards Initiative (MSI). Data transformation, alignment, and integrative analysis including correlation coefficient, partial least square (PLS) regression, one-way ANOVA, hierarchical clustering, and correlation network analysis were performed with the statistical software COVAIN ${ }^{75}$ under $^{-}$ MATLAB environment. The amount of each metabolite and bacteria OTUs were $\mathrm{z}$-scored across all samples. The correlation network associating metabolites (classified as MSI 1 and 2) and bacteria OTUs was constructed by Pearson's correlation coefficients (cutoff value $=0.8$ ). For network visualization, Girven-Newman algorithm ${ }^{76}$ was applied and visualization was performed with Cytoscape v3.7.2. (http://www.cytoscape.org/).

Concerning other data sets, the experimental groups were compared applying one-way ANOVA with Bonferroni post-hoc corrections for multiple testing. Where applicable, differences between two experimental groups were analyzed using Student's t-test with statistical significance threshold set at $p<0.05$. Each of the groups contained 8-10 biological replicates.

\section{Data availability}

The microbiota and metabolomics datasets generated during and analyzed during the current study are available in the European Nucleotide Archive [https://www.ebi.ac.uk/ena/browser/view/PRJEB37837] and MetaboLights repository, [www.ebi.ac.uk/metabolights/MTBLS1631] respectively.

Received: 19 August 2020; Accepted: 17 November 2020

Published online: 30 November 2020

\section{References}

1. Ferrecchia, C. E., Jensen, K. \& Van Andel, R. Intracage ammonia levels in static and individually ventilated cages housing C57BL/6 mice on 4 bedding substrates. J. Am. Assoc. Lab. Anim. Sci. 53, 146-151 (2014).

2. Jensen, T. L., Kiersgaard, M. K., Sorensen, D. B. \& Mikkelsen, L. F. Fasting of mice: a review. Lab. Anim. 47, 225-240 (2013).

3. Weindruch, R., Walford, R. L., Fligiel, S. \& Guthrie, D. The retardation of aging in mice by dietary restriction: longevity, cancer, immunity and lifetime energy intake. J. Nutr. 116, 641-654 (1986).

4. Masoro, E. J. Caloric restriction and aging: an update. Exp. Gerontol. 35, 299-305 (2000).

5. Duszka, K. et al. Complementary intestinal mucosa and microbiota responses to caloric restriction. Sci. Rep. 8, 11338 (2018).

6. Moen, B. et al. Effect of dietary fibers on cecal microbiota and intestinal tumorigenesis in azoxymethane treated $\mathrm{A} / \mathrm{J} \mathrm{min} /+$ mice. PLoS ONE 11, e0155402 (2016).

7. Hoover, W. H. \& Heitmann, R. N. Effects of dietary fiber levels on weight gain, cecal volume and volatile fatty acid production in rabbits. J. Nutr. 102, 375-379 (1972).

8. Drew, J. E. et al. Dietary fibers inhibit obesity in mice, but host responses in the cecum and liver appear unrelated to fiber-specific changes in cecal bacterial taxonomic composition. Sci. Rep. 8, 15566 (2018).

9. Wostmann, B. S., Bruckner-Kardoss, E. \& Knight, P. L. Jr. Cecal enlargement, cardiac output, and O2 consumption in germfree rats. Proc. Soc. Exp. Biol. Med. 128, 137-141 (1968).

10. Loesche, W. J. Accumulation of endogenous carbohydrate-containing compounds in the cecum of the germfree rat. Proc. Soc. Exp. Biol. Med. 131, 387-392 (1969).

11. Konishi, F., Oku, T. \& Hosoya, N. Hypertrophic effect of unavailable carbohydrate on cecum and colon in rats. J. Nutr. Sci. Vitaminol. (Tokyo) 30, 373-379 (1984).

12. Zijlstra, R. T., Jha, R., Woodward, A. D., Fouhse, J. \& van Kempen, T. A. Starch and fiber properties affect their kinetics of digestion and thereby digestive physiology in pigs. J. Anim. Sci. 90(Suppl 4), 49-58 (2012).

13. Ambery, A. G., Tackett, L., Penque, B. A., Hickman, D. L. \& Elmendorf, J. S. Effect of Corncob bedding on feed conversion efficiency in a high-fat diet-induced prediabetic model in C57Bl/6J mice. J. Am. Assoc. Lab. Anim. Sci. 53, 449-451 (2014).

14. Le Leu, R. K., Conlon, M. A., Bird, A. R. \& Clarke, J. M. Housing experimental rats in solid-based cages with digestible bedding may confound outcomes of nutritional studies. J. Sci. Food Agric. 95, 2155-2158 (2015).

15. Topping, D. L. \& Clifton, P. M. Short-chain fatty acids and human colonic function: roles of resistant starch and nonstarch polysaccharides. Physiol. Rev. 81, 1031-1064 (2001).

16. Macfarlane, S. \& Macfarlane, G. T. Regulation of short-chain fatty acid production. Proc. Nutr. Soc. 62, 67-72 (2003).

17. Tan, J. et al. The role of short-chain fatty acids in health and disease. Adv. Immunol. 121, 91-119 (2014).

18. Franklin, C. L. \& Ericsson, A. C. Microbiota and reproducibility of rodent models. Lab. Anim. (NY) 46, 114-122 (2017).

19. Schloss, P. D. Identifying and overcoming threats to reproducibility, replicability, robustness, and generalizability in microbiome research. mBio 9, e00525 (2018).

20. Gu, S. et al. Bacterial community mapping of the mouse gastrointestinal tract. PLoS ONE 8, e74957 (2013).

21. Zeng, H. et al. Integrating multiple analytical datasets to compare metabolite profiles of mouse colonic-cecal contents and feces. Metabolites 5, 489-501 (2015).

22. Fraumene, C. et al. Caloric restriction promotes rapid expansion and long-lasting increase of Lactobacillus in the rat fecal microbiota. Gut Microbes 9, 104-114 (2018).

23. Tanca, A. et al. Caloric restriction promotes functional changes involving short-chain fatty acid biosynthesis in the rat gut microbiota. Sci. Rep. 8, 14778 (2018).

24. Zhang, C. et al. Structural modulation of gut microbiota in life-long calorie-restricted mice. Nat. Commun. 4, 2163 (2013).

25. Liu, T. et al. A more robust gut microbiota in calorie-restricted mice is associated with attenuated intestinal injury caused by the chemotherapy drug cyclophosphamide. mBio 10, e02903 (2019). 
26. Wang, S. et al. Gut microbiota mediates the anti-obesity effect of calorie restriction in mice. Sci. Rep. 8, 13037 (2018).

27. Hervik, A. K. \& Svihus, B. The role of fiber in energy balance. J. Nutr. Metab. 2019, 4983657 (2019).

28. Slavin, J. L., Brauer, P. M. \& Marlett, J. A. Neutral detergent fiber, hemicellulose and cellulose digestibility in human subjects. J. Nutr. 111, 287-297 (1981).

29. Holloway, W. D., Tasman-Jones, C. \& Lee, S. P. Digestion of certain fractions of dietary fiber in humans. Am. J. Clin. Nutr. 31, 927-930 (1978).

30. Crawford, D. F., Anthony, W. B. \& Harris, R. R. Evaluation of concentrated hemicellulose extract as cattle feed. J. Anim. Sci. 46, $32-40$ (1978).

31. Niemi, P. et al. Interactions of a lignin-rich fraction from brewer's spent grain with gut microbiota in vitro. J. Agric. Food Chem. 61, 6754-6762 (2013).

32. Begum, A. N. et al. Dietary lignins are precursors of mammalian lignans in rats. J. Nutr. 134, 120-127 (2004).

33. Potgieter, F. J., Wilke, P. I., van Jaarsveld, H. \& Alberts, D. W. The in vivo effect of different bedding materials on the antioxidant levels of rat heart, lung and liver tissue. J. S. Afr. Vet. Assoc. 67, 27-30 (1996).

34. Buddaraju, A. K. \& Van Dyke, R. W. Effect of animal bedding on rat liver endosome acidification. Comp. Med. 53, 616-621 (2003).

35. National Research Council. Guide for the Care and Use of Laboratory Animals 8th edn. (National Academies Press, New York, 2011).

36. Sauer, M. B. et al. Clinical pathology laboratory values of rats housed in wire-bottom cages compared with those of rats housed in solid-bottom cages. J Am Assoc Lab Anim Sci 45, 30-35 (2006).

37. Giral, M., Garcia-Olmo, D. C. \& Kramer, K. Effects of wire-bottom caging on heart rate, activity and body temperature in telemetryimplanted rats. Lab. Anim. 45, 247-253 (2011).

38. Barnes, R. H., Kwong, E. \& Fiala, G. Effects of the prevention of coprophagy in the rat. III. Digestibility of protein and fat. J. Nutr. 65, 251-258 (1958).

39. Barnes, R. H., Tuthill, S., Kwong, E. \& Fiala, G. Effects of the prevention of coprophagy in the rat. V. Essential fatty acid deficiency. J. Nutr. 68, 121-130 (1959).

40. Barnes, R. H. \& Kwong, E. Choline biosynthesis and choline requirement in the rat as affected by coprophagy. J. Nutr. 92, 224-232 (1967).

41. Barnes, R. H. \& Fiala, G. Effects of the prevention of coprophagy in the rat. II. Vitamin B12 requirement. J. Nutr. 65, 103-114 (1958).

42. Barnes, R. H., Fiala, G. \& Kwong, E. Prevention of coprophagy in the rat and the growth-stimulating effects of methionine, cystine and penicillin when added to diets containing unheated soybeans. J. Nutr. 85, 127-131 (1965).

43. Barnes, R. H., Kwong, E. \& Fiala, G. Effects of the prevention of coprophagy in the rat. IV. Biotin. J. Nutr. 67, 599-610 (1959).

44. Kwong, E. \& Barnes, R. H. Role of coprophagy in masking dietary deficiencies of cystine in the rat. J. Nutr. 105, 1457-1465 (1975).

45. Schulze, J. \& Haenel, H. Relationships between coprophagia, intestinal flora and vitamins in experimental animals. Z. Versuchstierkd 11, 190-206 (1969).

46. Neale, R. J. Coprophagy in iron-deficient rats. Lab. Anim. 16, 204-207 (1982).

47. Ebino, K. Y., Yoshinaga, K., Saito, T. R. \& Takahashi, K. W. A simple method for prevention of coprophagy in the mouse. Lab. Anim. 22, 1-4 (1988).

48. Liu, T. W. et al. Nondigestible fructans alter gastrointestinal barrier function, gene expression, histomorphology, and the microbiota profiles of diet-induced obese C57BL/6J mice. J. Nutr. 146, 949-956 (2016).

49. Pan, F. et al. Predominant gut Lactobacillus murinus strain mediates anti-inflammaging effects in calorie-restricted mice. Microbiome 6, 54 (2018).

50. Zou, J. et al. Fiber-mediated nourishment of gut microbiota protects against diet-induced obesity by restoring IL-22-Mediated Colonic Health. Cell Host Microbe 23, 41-53 (2018).

51. Desai, M. S. et al. A dietary fiber-deprived gut microbiota degrades the colonic mucus barrier and enhances pathogen susceptibility. Cell 167, 1339-1353 (2016).

52. Weitkunat, K. et al. Effects of dietary inulin on bacterial growth, short-chain fatty acid production and hepatic lipid metabolism in gnotobiotic mice. J. Nutr. Biochem. 26, 929-937 (2015).

53. Chassaing, B. et al. Lack of soluble fiber drives diet-induced adiposity in mice. Am. J. Physiol. Gastrointest. Liver Physiol. 309, G528-541 (2015).

54. Knapp, B. K. et al. Soluble fiber dextrin and soluble corn fiber supplementation modify indices of health in cecum and colon of Sprague-Dawley rats. Nutrients 5, 396-410 (2013).

55. Vince, A. J., McNeil, N. I., Wager, J. D. \& Wrong, O. M. The effect of lactulose, pectin, arabinogalactan and cellulose on the production of organic acids and metabolism of ammonia by intestinal bacteria in a faecal incubation system. Br. J. Nutr. 63, 17-26 (1990).

56. Stark, A. H. \& Madar, Z. In vitro production of short-chain fatty acids by bacterial fermentation of dietary fiber compared with effects of those fibers on hepatic sterol synthesis in rats. J. Nutr. 123, 2166-2173 (1993).

57. Chassard, C., Delmas, E., Robert, C. \& Bernalier-Donadille, A. The cellulose-degrading microbial community of the human gut varies according to the presence or absence of methanogens. FEMS Microbiol. Ecol. 74, 205-213 (2010).

58. Wedekind, K. J., Mansfield, H. R. \& Montgomery, L. Enumeration and isolation of cellulolytic and hemicellulolytic bacteria from human feces. Appl. Environ. Microbiol. 54, 1530-1535 (1988).

59. Robert, C. \& Bernalier-Donadille, A. The cellulolytic microflora of the human colon: evidence of microcrystalline cellulosedegrading bacteria in methane-excreting subjects. FEMS Microbiol. Ecol. 46, 81-89 (2003).

60. Betian, H. G., Linehan, B. A., Bryant, M. P. \& Holdeman, L. V. Isolation of a cellulotytic Bacteroides sp. from human feces. Appl Environ Microbiol 33, 1009-1010 (1977).

61. Neis, E. P., Dejong, C. H. \& Rensen, S. S. The role of microbial amino acid metabolism in host metabolism. Nutrients 7, 2930-2946 (2015).

62. Cheng, W. et al. Effects of a galacto-oligosaccharide-rich diet on fecal microbiota and metabolite profiles in mice. Food Funct. 9, 1612-1620 (2018).

63. Han, J., Meng, J., Chen, S. \& Li, C. Integrative analysis of the gut microbiota and metabolome in rats treated with rice straw biochar by $16 \mathrm{~S}$ rRNA gene sequencing and LC/MS-based metabolomics. Sci. Rep. 9, 17860 (2019).

64. Tang, Z. Z. et al. Multi-omic analysis of the microbiome and metabolome in healthy subjects reveals microbiome-dependent relationships between diet and metabolites. Front. Genet. 10, 454 (2019).

65. Tofalo, R., Cocchi, S. \& Suzzi, G. Polyamines and gut microbiota. Front. Nutr. 6, 16 (2019).

66. Klymiuk, I. et al. The human gastric microbiome is predicated upon infection with Helicobacter pylori. Front. Microbiol. 8, 2508 (2017).

67. Afgan, E. et al. The Galaxy platform for accessible, reproducible and collaborative biomedical analyses: 2018 update. Nucleic Acids Res. 46, W537-W544 (2018).

68. Callahan, B. J. et al. DADA2: High-resolution sample inference from Illumina amplicon data. Nat. Methods 13, 581-583 (2016).

69. Yilmaz, P. et al. The SILVA and "All-species Living Tree Project (LTP)" taxonomic frameworks. Nucleic Acids Res. 42, D643-648 (2014).

70. Katoh, K., Misawa, K., Kuma, K. \& Miyata, T. MAFFT: a novel method for rapid multiple sequence alignment based on fast Fourier transform. Nucleic Acids Res. 30, 3059-3066 (2002). 
71. Price, M. N., Dehal, P. S. \& Arkin, A. P. FastTree 2-approximately maximum-likelihood trees for large alignments. PLoS ONE 5, e9490 (2010).

72. Weckwerth, W., Wenzel, K. \& Fiehn, O. Process for the integrated extraction, identification and quantification of metabolites, proteins and RNA to reveal their co-regulation in biochemical networks. Proteomics 4, 78-83 (2004).

73. Obermeyer, G., Fragner, L., Lang, V. \& Weckwerth, W. Dynamic adaption of metabolic pathways during germination and growth of lily pollen tubes after inhibition of the electron transport chain. Plant Physiol. 162, 1822-1833 (2013).

74. Doerfler, H. et al. Granger causality in integrated GC-MS and LC-MS metabolomics data reveals the interface of primary and secondary metabolism. Metabolomics 9, 564-574 (2013).

75. Sun, X. \& Weckwerth, W. COVAIN: a toolbox for uni- and multivariate statistics, time-series and correlation network analysis and inverse estimation of the differential Jacobian from metabolomics covariance data. Metabolomics 8, 81-93 (2012).

76. Girvan, M. \& Newman, M. E. Community structure in social and biological networks. Proc. Natl. Acad. Sci. USA 99, 7821-7826 (2002)

\section{Acknowledgements}

Open access funding provided by the University of Vienna.

\section{Author contributions}

A.G. performed animal experiments, samples and data analysis as well as contributed to the manuscript writing; L.F. performed GC-MS metabolites analysis; S.T. and W.L. performed the bioinformatical analysis of microbiota composition; W.L. analyzed GC-MS experiments data; X.L. provided statistical analysis tool and methods; W.W. contributed with metabolomics platform, expert advice and supervised metabolomics analysis; J.K. contributed expert advice; K.D. designed and performed the experiments, analyzed samples and wrote the manuscript. All authors approved the final manuscript.

\section{Competing interests}

The authors declare no competing interests.

\section{Additional information}

Supplementary information is available for this paper at https://doi.org/10.1038/s41598-020-77831-3.

Correspondence and requests for materials should be addressed to K.D.

Reprints and permissions information is available at www.nature.com/reprints.

Publisher's note Springer Nature remains neutral with regard to jurisdictional claims in published maps and institutional affiliations.

(i) Open Access This article is licensed under a Creative Commons Attribution 4.0 International License, which permits use, sharing, adaptation, distribution and reproduction in any medium or format, as long as you give appropriate credit to the original author(s) and the source, provide a link to the Creative Commons licence, and indicate if changes were made. The images or other third party material in this article are included in the article's Creative Commons licence, unless indicated otherwise in a credit line to the material. If material is not included in the article's Creative Commons licence and your intended use is not permitted by statutory regulation or exceeds the permitted use, you will need to obtain permission directly from the copyright holder. To view a copy of this licence, visit http://creativecommons.org/licenses/by/4.0/.

(c) The Author(s) 2020 